\title{
DETC2004-57046
}

\section{ROBOTIC SYSTEM TO EVALUATE FORCE FEEDBACK IN MINIMALLY INVASIVE COMPUTER AIDED SURGERY}

\author{
Hermann Mayer, István Nagy and Alois Knoll \\ Department of Robotics and Embedded Systems \\ Technische Universität München \\ 85748 Garching \\ Email: \{mayerh,nagy,knoll\}@in.tum.de
}

\author{
Eva Schirmbeck and Robert Bauernschmitt \\ Klinik für Herz- und Gefässchirurgie \\ Deutsches Herzzentrum München \\ 80636 München \\ Email: \{schirmbeck,bauernschmitt\}@dhm.mhn.de
}

\begin{abstract}
We present an experimental system for robot assisted, minimally invasive surgery that is capable of force measurement and haptic feedback. While minimally invasive surgery with robots provides several advantages like reduced tissue trauma and shorter recovery times, there are also some inherent shortcomings. Referring to surgeons, the most significant issue is the lack of haptic feedback. This often causes collateral trauma and leads to prolonged operation time. Therefore we have developed an open platform, which combines the advantages of present systems with the possibility of force reflection. We have included features known from commercial available systems, like full Cartesian control of the end effector, stereo vision and ergonomic input devices. We used the system to perform basic surgical tasks (like knot-tying) and to evaluate force feedback.
\end{abstract}

\section{INTRODUCTION}

During the last decade minimally invasive surgery has become the leading method for many surgical interventions. Unlike open surgery, minimally invasive surgery only needs small incisions in the patient's body. This leads to a drastic reduction of tissue trauma and therefore to shorter recovery times. In the beginning, this technique was performed manually with specialized instruments. Surgeons had to cope with restricted manipulability of the end-effector and poor visual feedback. These drawbacks were overcome by employment of dedicated robotic systems. Commercially available telemanipulators like the daVinci workstation [1] or the ZEUS system [2,3] provide a decoupling of input device and instruments. The surgeon controls the instruments with a master console that is placed separately in the operating room. The instructions are carried out by a telemanipulator, whose end-effectors perform the operations. This allows comfortable work and full 6 degree-of-freedom (DOF) control of the instruments. Additionally, vision of the surgeon is improved by means of endoscopic stereo cameras, whose images are displayed at the surgeon's console. This system has a proven record, and many delicate operations have been performed [4-7]. A number of similar systems, both in research and for commercial use have been developed. These include, for example, a robotic system developed at UC Berkeley, which has already been used to perform certain surgical tasks like suturing and knot-tying [8]. The Korean Advanced Institute of Science and Technology has developed a micro-telerobot system that provides force feedback [9]. In Germany the first systems for robotic surgery was built at the Research Facility in Karlsruhe [10] and DLR [11]. While the former system provides no force feedback, the DLR system is equipped with PHANToM(tm) devices for haptic display. Despite the advantages the systems offer, there are also needs of surgeons that have not been met. The most crucial issue is the lack of sensitive force feedback combined with delicate and fine instruments [12-14]. This often leads to unpleasant side effects like damaging thread material or even lacerating healthy tissue. It is in particular this shortcoming that results in fatigue of the operator, due to visual compensation of the missing haptic feedback.

Incorporation of force feedback in systems for robotic surgery is therefore a crucial factor in improving reaction to tis- 


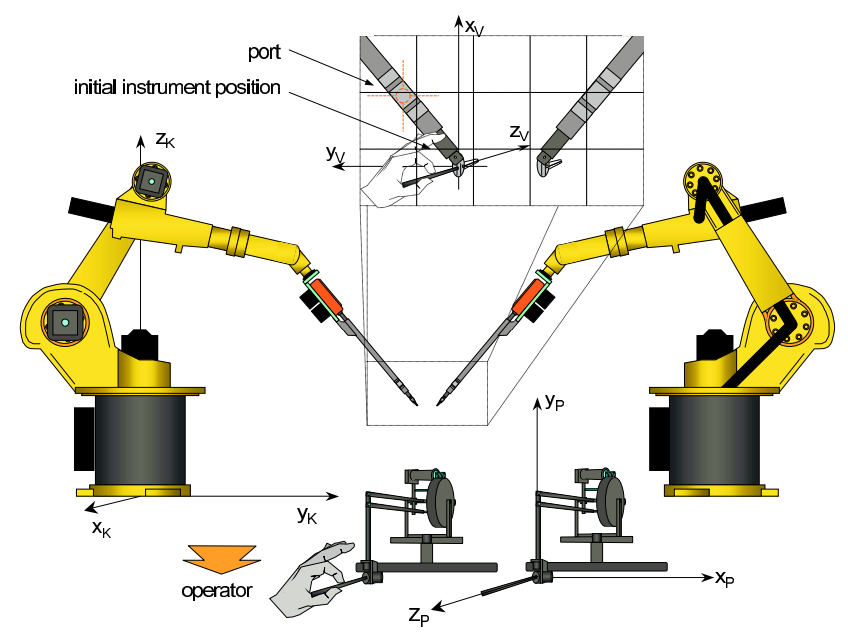

Figure 1. SYSTEM SET-UP

sue contact. Our aim is to provide the surgeon with an operation environment very similar to manual instrumental surgery (i.e. the surgeon can always feel forces exerted on the instruments). According to [15], the influence of force feedback on operation time seems to be even more important than it is for visual feedback. Following this analysis of deficiencies, we developed an open evaluation platform for robotic surgery that was tailored to the needs of sensitive force feedback for delicate operations like bypass operations in cardiac surgery (Fig. 1). Our workstation is not a telemanipulator that is controlled by visual servoing of the surgeon. Instead, it can be directly controlled by transmitting 6 DOF coordinates to its control unit. This is an important feature for closing control loops in machine learning applications, which can be applied in order to autonomously perform certain recurrent tasks, e.g. automated cutting or knot-tying.

\section{METHODS AND MATERIALS}

Similar to other systems, our setup comprises an operatorside master console for in-output and a patient-side robotic manipulator that directly interacts with the operating environment. As shown in Fig. 1, our system has two manipulators, which are controlled by two input devices. Each of the two arms of our surgical robot is composed of the following subsystems. A lowpayload robot, which bears a surgical instrument that is deployed with the surgical workstation daVinci (TM). We have developed a special adapter that interconnects the robot's flange with the instrument. The surgical instruments have three degrees of freedom. A micro-gripper at the distal end of the shaft can be rotated and adaptation of pitch and yaw angles is possible. Since the yaw angle of each of the two fingers of the gripper can be controlled separately to open and close the gripper. All movable parts of the gripper are driven by steel wires. Their motion is controlled by four driving wheels at the proximal end of the instrument, one for each degree of freedom (two for yaw of the fingers). In order to control the instrument, we have flanged servos to each driving wheel by means of an Oldham coupling. This guarantees instrument movement free of jerk. The servo controllers are connected via serial lines to a multi-port interface card. Since the rotation of the robot's flange and the rotation of the instrument share one axis, the combination of robot and instrument results in a manipulator with eight degrees of freedom. That means our system is a redundant manipulator. This can be exploited to evaluate different kinematical behaviors. The most important one is trocar kinematics. This allows 6 dof control of the end effector, while the shaft of the instrument has to be moved about a fixed fulcrum (keyhole surgery). Position and orientation of the manipulators are controlled by two PHANToM devices (Fig. 1). This device is available in different versions with different capabilities. Our version provides a full 6 dof input, while force feedback is restricted to three translational directions. The user controls a stylus pen that is equipped with a switch that can be used to open and close the micro-grippers.

\section{Force Feedback}

The most interesting feature of the PHANToM devices we used, is their capability of providing the user with haptic feedback. Forces are feeded back by small servo motors incorporated in the device. They are used to steer the stylus pen in a certain direction. This creates the impression of occurring forces, while the user is holding the pen at a certain posture. The force sensors were applied directly on the shaft of the instrument. Since the shaft of the surgical instrument is made of carbon fibre, force sensors have to be very sensitive and reliable. Therefore we decided to apply strain gauge sensors, which are employed for industrial force registration. As shown in Fig. 2, the sensor gauges are applied at the distal end of the instrument's shaft, i.e. near the gripper. At the top of Fig. 2, one can see the perpendicular arrangement of strain gauges as full bridges. One full bridge of sensors is used for each direction. The signals from the sensors are amplified and transmitted via CAN-bus to a PC system. Sensor readings are blurred with noise, hence we have applied digital filters to stabilize the results. Since we know the position and orientation of the instruments, we can transform occurring forces back to the coordinate system of the PHANToM devices. Therefore the user has the impression of direct haptic immersion.

\section{Trocar Kinematics}

The basic idea of minimally invasive surgery is, that only small openings have to be made into the surface of the patient's thorax (so-called keyholes, Fig. 3). That means the translational movements of the instruments are essentially restricted by shifts and rotations about these holes. In order to provide the surgeon with a comfortable environment, it is desirable to map the move- 


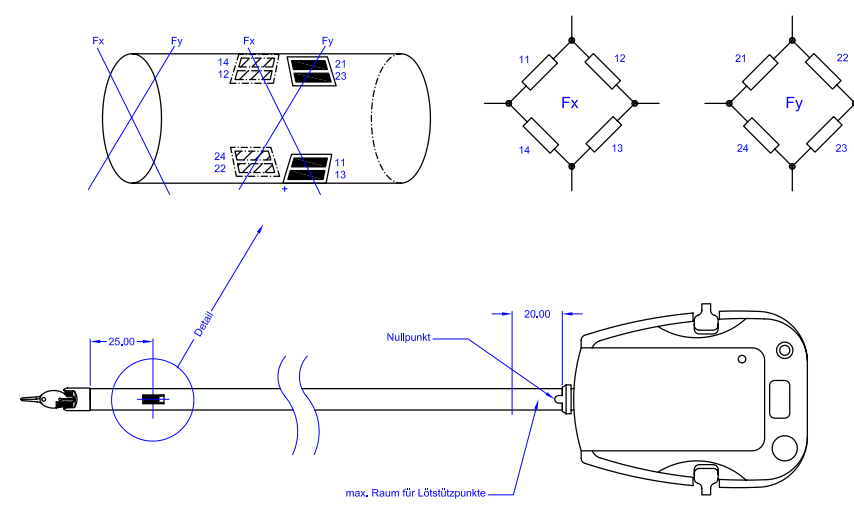

Figure 2. APPLICATION OF STRAIN GAUGES TO AN INSTRUMENT

ments of the stylus at the input device directly to instrument motions. Therefore we have to consider the inverse kinematics of our system. That means we have to find a mapping of an arbitrary posture of the instrument's tip to a position of the motors that control the eight degrees of freedom.

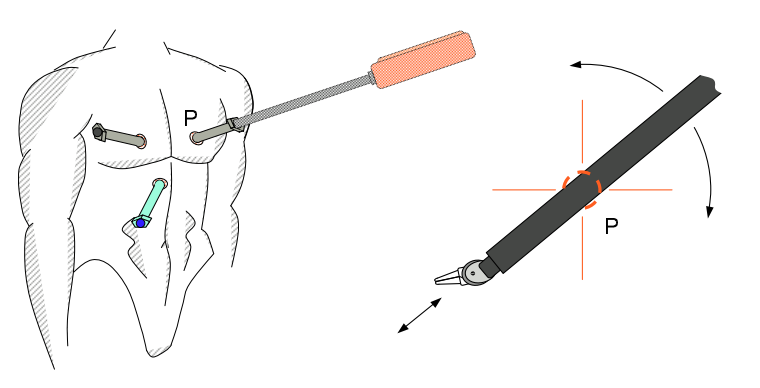

Figure 3. TROCAR POINT KINEMATICS

The desired position of the instrument is given by the position of the input stylus. It is represented by a homogenous transform matrix. Since the position of the instrument's shaft is restricted by the port (the position of the keyhole), there is only one possibility for aligning the instrument. The angle of the corresponding joints of the instrument can be found by geometric considerations. For result, we get the position of the instrument's shaft. As this axis is identical to the flange axis of the robot, we have got the position of the flange. Given this information, we now can determine the backwards kinematics of the robot. This is a standard procedure, whose detailed calculation will be neglected here. As a final result we can implement a mapping from the position of the input stylus to the position of the instrument. That means the surgeon is provided with a direct remote control of the surgical instruments. A detailed description of the com- plex trocar kinematics can be found in [16].

\section{System Features}

We give only a short list of system features we think to be important. The use of commercially available subsystems (robots, instruments, amplifiers) guarantees reliability and simplifies mass-production at comparable low prices. Particular advantages of this setup with multi-purpose robots are high precision and stiffness, moderate costs and an advanced dynamic behavior. The latter could be exploited to perform advanced tasks in motion compensation (e.g. support for beating heart surgery as it was proposed in [17], or compensation for respiratory motion of the ribs). The modular character of this setup simplifies the adaptation of the system to technical improvements (e.g. new surgical instruments). Another advantage is the fact that our manipulator is a robot under Cartesian control whose position can be controlled precisely. Finally, the most important feature is the possibility for evaluation of force feedback in combination with endoscopic vision in robotic surgery. In order to make navigation easier, we additionally equipped the system with an endoscopic stereo camera system to observe the operation environment.

\section{EXPERIMENTAL RESULTS}

The original setup of the system, as we have used it for our first evaluations, can be seen in Fig. 4. We have performed several tasks adopted from surgical practice with this system. Our special emphasis was on surgical knot-tying.

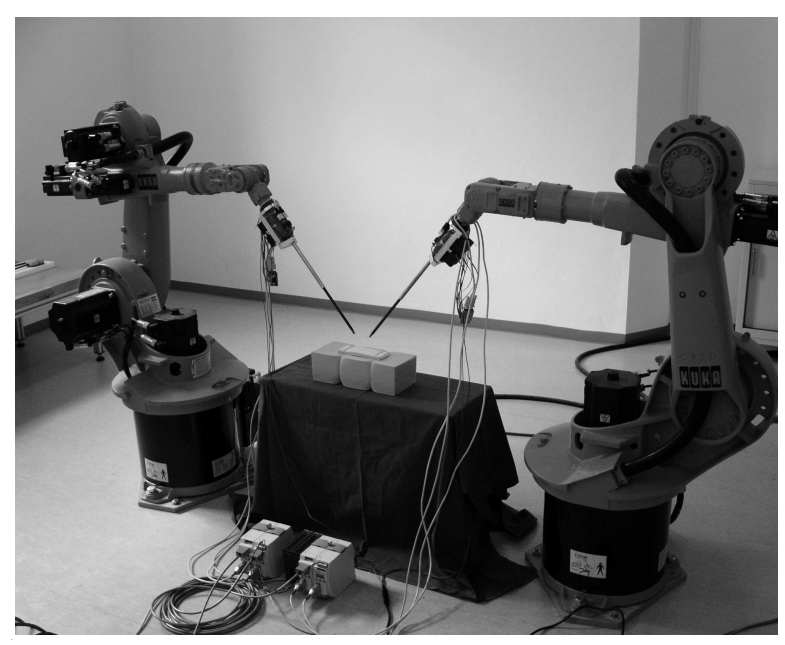

Figure 4. EXPERIMENTAL SETUP OF THE SYSTEM

We successfully have made several different knots on a block of Styrofoam. We experienced some difficulties, because at this time navigation was only based on the image of a single 
camera. The displayed images are not capable of providing realistic depth information. This experience has also been made by other authors (e.g. [17]). Therefore we are now using a stereo camera system. Even with monocular view, knot tying was performed in a time that is acceptable for a first experimental evaluation (approx. one minute per knot). Force feedback has provided very realistic impressions of the environment. Forces were displayed in correct relation and along the right direction of the input device. Haptic feedback has completely prevented destruction of thread material or damaging Styrofoam. Force feedback turned out to be helpful when making contacts of the instruments with manipulated objects.

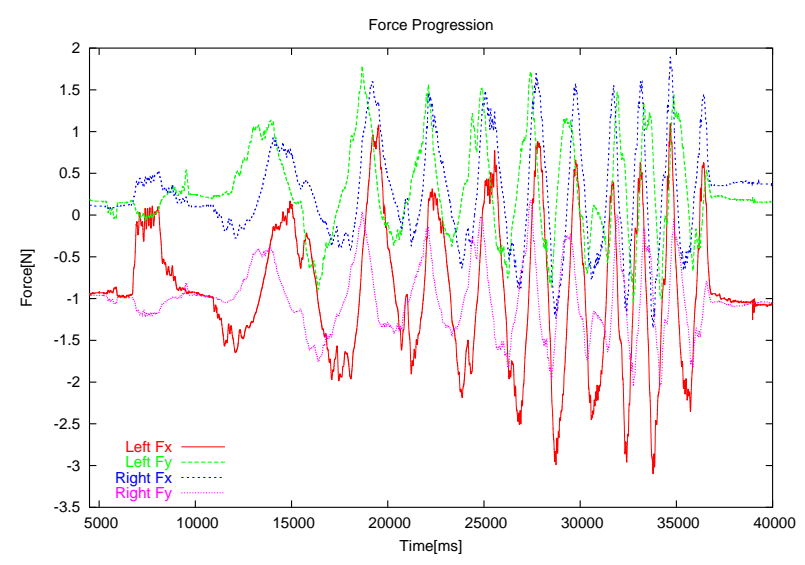

Figure 5. Winding a thread to make loops

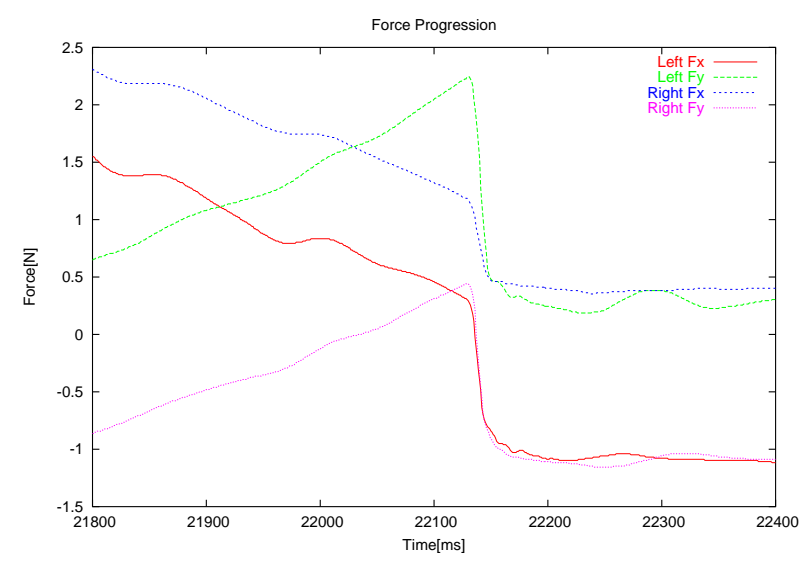

Figure 6. Breaking the thread during winding

\section{Winding}

The first operation sequence we evaluated was winding thread during knot tying. Forces are acquired only in the $X Y$ Plane perpendicular to the instrument shaft, as our current setup does not yet allow the measurement of forces along the shaft. Winding thread to form loops is a subtask in instrumental knot tying (cf. [18]) and if executed by a surgeon, only very low forces arise, since a human operator easily copes with this task using only visual feedback. However in robot assisted surgery scenarios high fidelity force sensory is indispensable, as the visual modality is very difficult to interpret. Accordingly, robotic winding can be accomplished only in a force-controlled manner. On the one hand forces are preferably to be kept constant, on the other hand suture break must be avoided. Fig. 5 shows the force progression during a winding process. The frequency of force peeks in a certain direction grows, as the suture material gets shorter. Nevertheless the forces are quite constant during the whole manipulation. Figure 6 shows a magnified view of an accidental break of the thread during a further winding process. Due to the high time resolution $(1 \mathrm{~ms})$ the instant recognition of such suture breaks is possible, preventing the robotic system from unexpected behavior.

\section{Preventing Suture Material Damage}

The tensile strength of absorbable and non-absorbable sutures is critical both during and after surgical procedures. Breaking strength can be measured using either a "straight pull" test or a "knot pull" test. Having the breaking strengths of all used sutures enables us to prevent suture material damage by limiting the applicable forces to adequate maximal values. Fig. 7 shows the progression of forces while trying to break original surgical suture material, in this case Ethicon PROLENE (7/0, Polypropylen, not absorbable). Fig. 8 shows breaking the thread (PROLENE 7/0) while tying a knot. As expected, the thread was broken at the knot position by significantly less force impact.

\section{Collision Detection}

Avoiding the collision of the instruments in robot assisted minimally invasive surgery is not an easy task. Therefore a symbolic representation of the whole robotic system, including both the instruments and the arms, were necessary. Furthermore exact position control and a collision detection software subsystem are indispensable. Most setups however do not provide the above mentioned infrastructure. A human operator will easily avoid instrument collisions, but in an autonomous mode other solutions are necessary. A force controlled setup will not prevent collisions, but an early detection can avoid damaging the instruments. Figure 9 shows the forces recorded while an instrument collision, the instrument velocities were within ranges typical to this scenario. We observe, that the highest peak ( $Y$-force component of the left instrument) arises in approximately $35 \mathrm{~ms}$. With a robot 


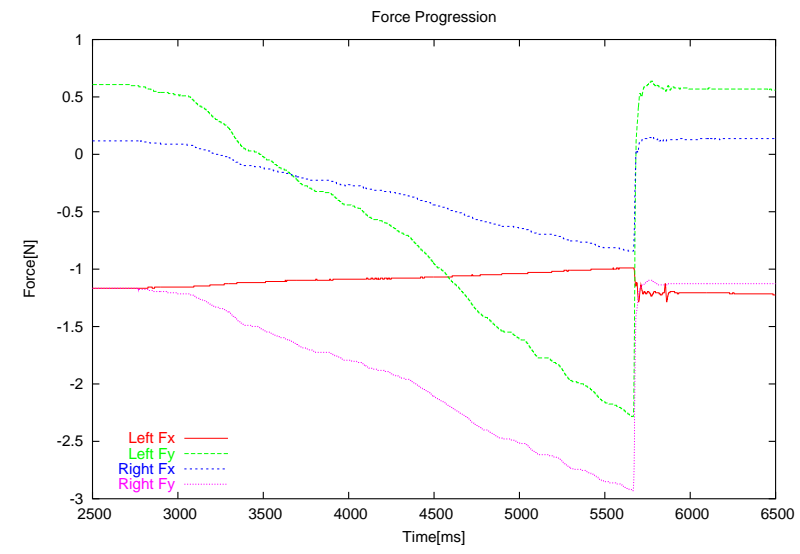

Figure 7. Breaking Ethicon $7 / 0$ by normal pulling

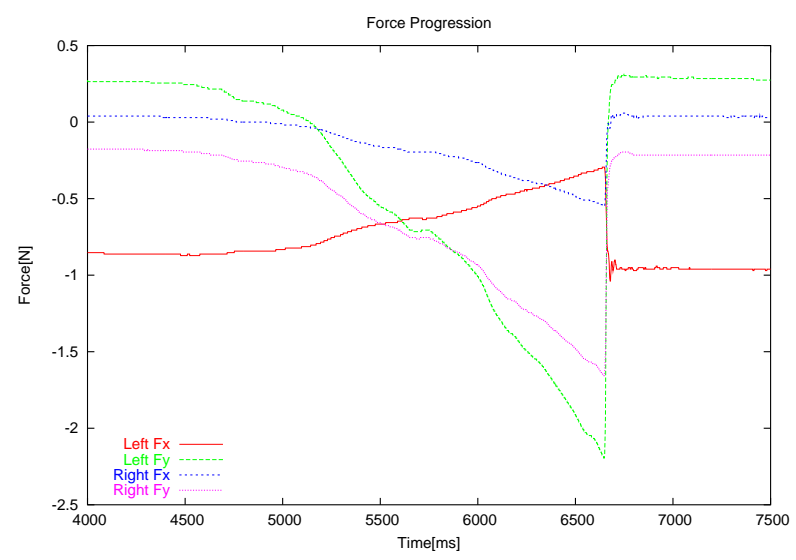

Figure 8. Breaking Ethicon 7/0 during knot tying

arm interpolation of $12 \mathrm{~ms}$ there are nearly 3 interpolation periods to react when such a situation appears, providing a satisfactory collision interception.

\section{CONCLUSION}

We have presented a novel approach of a robotic system for minimally invasive surgery. It is mainly composed of commercially available subsystems. This has several advantages like precision, reliability and a good dynamic behavior. The main purpose of the system is evaluation of force feedback. We found out that performance of certain surgical tasks like knot tying will massively profit from this feature. Forces are measured at the surgical instruments and feeded back into the surgeon's hands using multi-dimensional haptic styluses. For future evaluation we are planning long-term tests to find out if force feedback can prevent surgeon's fatigue. The current arrangement of input devices, however, is not very comfortable. Therefore we are planning to test different rearrangements of

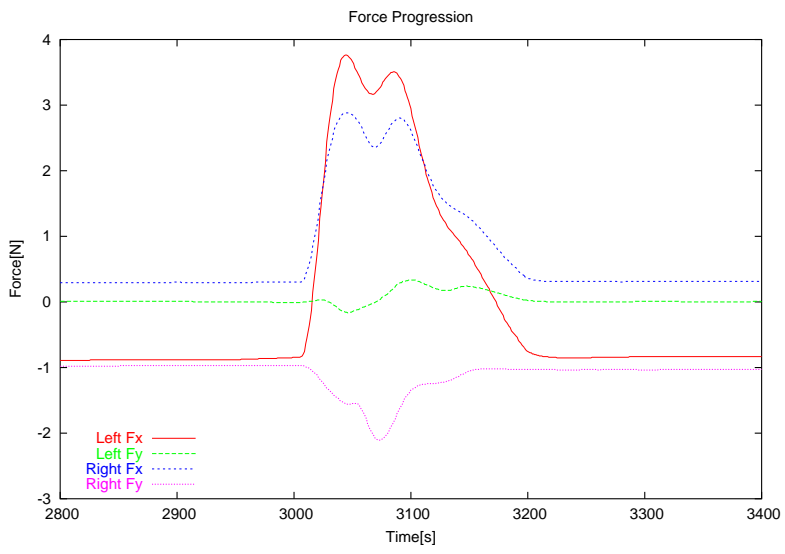

Figure 9. Colliding instruments

this setup and to develop an own input instrument to replace the stylus pen.

Integration of force feedback with stereo vision, as offered by the system, will improve accuracy, drastically reduce the time needed for operations and tissue trauma, along with a reduction of stress on the surgeon. This could lead to a wider acceptance of robotic surgery by both, patients and surgeons. The system's software interface and mechanical set-up descriptions are freely available to enable other research groups to participate in the development.

\section{REFERENCES}

[1] Guthart, G., and Salisbury, J., 2000. "The intuitive telesurgery system: Overview and application". IEEE ICRA, San Francisco, pp. 618-621.

[2] A. Garcia-Ruiz, N. G. S. e. a., 1997. "Robotic surgical instruments for dexterity enhancement in thoracoscopic coronary artery bypass graft". J Laparoendosc Adv Surg Tech, 7 (5), pp. 277-283.

[3] D. Boehm, H. R. e. a., 2000. "Clinical use of a computerenhanced surgical robotic system for endoscopic coronary artery bypass grafting on the beating heart". Thorac Cardiov Surg, 48 , pp. 198-202.

[4] R. Bauernschmitt, H. M., and Lange, R., 2001. "Telemanipulators in cardiac surgery: towards minimally invasive operations on the heart". Advances in Interactive Multimodal Telepresence Systems, pp. 125-133.

[5] F. Mohr, V. Falk, A. D. e. a., 2001. "Computer enhanced robotic cardiac surgery: Experience in 148 patients". The Journal of Thoracic and Cardiovascular Surgery, 121 (5), pp. 842-853.

[6] U. Kappert, R. Cichon, J. S. e. a., 2000. "Closed-chest coronary artery surgery on the beating heart with the use of 
a robotic system". The Journal of Thoracic and Cardiovascular Surgery, 120 (4), pp. 809-811.

[7] H. Shennib, A. Bastawisy, M. M., and Moll, H., 1998. "Computer assisted telemanipulation: an enabling technology for endoscopic coronary artery bypass". Annals of Thoracic Surgery, 66 (3), pp. 1060-1063.

[8] M. Cavusoglu, F. Tendick, M. C., and Sastry, S., 2003. "Robotics for telesurgery: Second generation berkeley/ucsf laparoscopic telesurgical workstation and looking towards the future applications". Industrial Robot, Special Issues on Medical Robotics, 30 (1) .

[9] D. Kwon, K. Woo, S. S. e. a., 1998. "Microsurgical telerobot system". IEEE/RSJ International Conference on Intelligent Robots and Systems , pp. 945-950.

[10] U. Voges, E. Holler, B. N. e. a., 1997. "Evaluation of artemis: the advanced robotics and telemanipulator system for minimally invasive surgery". Proceedings IARP 2nd Workshop on Medical Robotics, pp. 137-148.

[11] G. Wei, K. A., and Hirzinger, G., 1997. "Real-time visual servoing for laparoscopic surgery". IEEE Engineering in Medicine and Biology, 16 (1), pp. 40-45.

[12] L. Panait, C. D., and Merrell, R., 2002. "Applications of robotics in surgery". Chirurgica (Bucur), 97 (6) , pp. 549555.

[13] V. Urban, M. Wapler, J. N. e. a., 1998. "Robot-assisted surgery system with kinesthetic feedback". Computer Aided Surgery (CAS), 3 (4), pp. 205-209.

[14] J. Bowersox, P. C., and Porta, A. L., 1998. "Use of an intuitive telemanipulator system for remote trauma surgery: an experimental study". J Am Coll Surg, 186 (6), pp. 615621.

[15] J. Thompson, M. O., and Sheridan, T., 1999. "Human factors in telesurgery: effects of time delay and asynchrony in video and control feedback with local manipulative assistance". Telemed Journal, 5 (2), pp. 129-137.

[16] H. Mayer, I. N., and Knoll, A., 2004. "Inverse kinematics of a manipulator for minimally invasive surgery". Technical Report, Technische Universitaet Muenchen .

[17] T. Ortmaier, M. G., and Hirzinger, G., 2002. "Robust motion estimation in robotic surgery on the beating heart". Computer Assisted Radiology and Surgery (CARS) .

[18] C. Cao, C. M., and Payandeh, S., 1996. "Task and motion analyses in endoscopic surgery". Proceedings ASME Dynamic Systems and Control Division , pp. 583-590. 